Article

\title{
Fabrication and Performance of Composite Microencapsulated Phase Change Materials with Palmitic Acid Ethyl Ester as Core
}

\author{
Qing Yin, Zhenguo Zhu, Wei Li *, Maolian Guo, Yu Wang, Jianping Wang and Xingxiang Zhang * \\ Tianjin Key Laboratory of Advanced Fibers and Energy Storage, School of Material Science and Engineering, \\ Tianjin Polytechnic University, Tianjin 300387, China; yinqing02@163.com (Q.Y.); \\ zhuzhenguotjpu@163.com (Z.Z.); guomaoliansx@163.com (M.G.); wangyu_nankai@163.com (Y.W.); \\ jpwangcn@gmail.com (J.W.) \\ * Correspondence: hiweilee@gmail.com (W.L.); zhangpolyu@aliyun.com (X.Z.); \\ Tel.: +86-022-8395-5429 (W.L. \& X.Z.); Fax: +86-022-8395-5282 (W.L. \& X.Z.)
}

Received: 1 June 2018; Accepted: 29 June 2018; Published: 2 July 2018

\begin{abstract}
Microencapsulation of phase change materials (PCMs) could prevent the leakage of PCMs during solid-liquid phase change process. However, their applications are mainly limited by the compactness and thermal stability of the traditional polyurea shell microcapsules. To increase the thermal compactness and thermal stability of PCM microcapsules, tetraethylorthosilicate (TEOS) was employed to form polymer $/ \mathrm{SiO}_{2}$ composite shells to enhance the mechanical performance of polyurea and polyurethane microcapsule via interfacial polymerization and in situ polymerization. The morphology and chemical components of the microcapsules were characterized by field-emission scanning electron microscope (FE-SEM) and Fourier transform infrared (FT-IR) spectroscopy, respectively. The thermal properties of the microcapsules were investigated by differential scanning calorimetry (DSC) and thermal gravity analysis (TGA). The results showed the smoothness and compactness of both polyurea- $\mathrm{SiO}_{2}$ and polyurethane- $\mathrm{SiO}_{2}$ microcapsules enhanced slightly, when compared with that without TEOS addition. Moreover, the $\mathrm{SiO}_{2}$ composite shell had good effect on thermal compactness, as the weight loss rate of polyurea- $\mathrm{SiO}_{2}$ microcapsules and polyurethane- $\mathrm{SiO}_{2}$ microcapsules decreased $3.5 \%$ and $4.1 \%$, respectively.
\end{abstract}

Keywords: phase change material; microcapsules; polyurea; $\mathrm{SiO}_{2}$ nanoparticles; polyurethane

\section{Introduction}

Due to the rapid growth of the consumption of fossil fuels followed by environmental impact and energy resources [1], both scientific societies and industrial communities are concentrating on the improvement of energy utilization efficiency and the development of renewable energy [2-8]. Phase change materials (PCMs) are different from the conventional thermal energy-storage materials, for their capability in absorbing and releasing latent heat through phase transitions almost without temperature changing [9-15], and are considered as candidates of renewable energy materials [16-20].

Microencapsulation is a widely-used technique to encapsulate PCMs in the energy storage field. Microencapsulated phase change materials (MicroPCMs) have been studied since the late 1970s, because of their large storage capacity and isothermal nature of the storage process. The main advantages of MicroPCMs are as follows: (1) avoid the leakage of PCMs and isolate PCMs from matrix; (2) increase the heat transfer area of PCMs and improve their performance of thermal conductivity; (3) alter the decentralized state of PCMs and solve the problem that PCMs are incompatible with the surrounding medium in thermodynamics. However, traditional MicroPCMs still have some disadvantages of leakage during the solid-liquid phase transition [21,22], which probably results in 
some negative effects, such as gradual failure of energy storage, environment pollution and security potential danger. These disadvantages greatly limit practical thermal applications of MicroPCMs [23]. The conventional MicroPCMs with organic polymer shells have been widely studied, which possess good thermal stability but poor in thermal conductivity [24], such as urea formaldehyde resin, melamine formaldehyde resin, poly (methyl methacrylate) and polystyrene [25].

The general preparation methods of microcapsules are classified as physic-mechanical, physic-chemical, and chemical methods. The microcapsules prepared via physic-mechanical method generally have large particle size, rough surface, and low thermal storage capacity. In addition, microcapsules prepared via physic-mechanical method could obtain heat capacities of as high as about $145.2 \mathrm{~J} \cdot \mathrm{g}^{-1}$. However, it would lead to great difficulty with the method for processing at scale. The chemical methods, especially in situ polymerization or interfacial polymerization, are widely used for the preparation of microcapsules. In situ polymerization of amino resins, such as melamine formaldehyde (MF) resin and urea formaldehyde (UF) resin, are usually selected as the shell of MicroPCMs [25,26]. However, this method generally results in formaldehyde residue in microcapsules shells inevitably, so polyurethane and polyurea are two optimally used polymers to fabricate MicroPCMs [26]. The polyurea shell is usually prepared by aromatic isocyanates and amine, such as diphenylmethanediisocyanate (MDI), toluene diisocyanate (TDI) [27], but aromatic isocyanates can hydrolyze into highly toxic phenylamine. Aliphatic isophoronediisocyanate (IPDI) as monomer to replace aromatic isocyanates can form polyurea microcapsules with good yellowing resistance and without any phenylamine [28]. In addition, during the fabrication of polyurea, MicroPCMs, the toxic cosolvent tetrahydrofuran, acetone, and cyclohexane are usually chosen to form the miscibility of $n$-alkane PCMs and isocyanate. To solve these issues, ethyl palmitate has been used, and avoids the malodor in traditional MicroPCMs. In the end, certain defects exist in the thermal stability and compactness of traditional polyurea MicroPCMs.

In this paper, both polyethylene glycol 400 (PEG 400) and tetraethylorthosilicate (TEOS) were selected as functional shell-forming monomers to enhance the thermal stability and compactness of traditional polyurea MicroPCMs. PEG 400 has a macromolecule flexible chain, which can improve the flexible and resilient of polymer, while TEOS carry out hydrolysis polymerization to form polyurea- $\mathrm{SiO}_{2}$ or polyurethane- $\mathrm{SiO}_{2}$ composite shell. Therefore, it is anticipated that, the compactness and thermal stability would be enhanced via incorporating $\mathrm{SiO}_{2}$ into organic polyurea or polyurethane shell.

\section{Experimental Section}

\subsection{Materials}

Ethyl palmitate (99 wt \%) was purchased from Sinopharm Chemical Reagent Co., Ltd. (Beijing, China). Polyethylene glycol 400 (PEG400) and tetraethylorthosilicate (TEOS, 99 wt \%) were purchased from Aladdin (Shanghai, China). Isophoronediisocyanate (IPDI, 99 wt \%) and diethylenetriamine (DETA, 99 wt \%) were obtained from Adamas Reagent Co., Ltd (Shanghai, China). Styrene-maleic anhydride copolymer solution (SMA) employed as surfactant was kindly supplied by Institute of Functional Fiber, Tianjin Polytechnic University (Tianjin, China).

\subsection{Microencapsulation Process}

\subsubsection{Fabrication of $\mathrm{SiO}_{2}-$ Polyurea Microcapsules}

The microcapsules were synthesized by the interfacial polymerization: SMA ( $5 \mathrm{~g})$ was dissolved into deionized water and formed the aqueous phase. Ethyl palmitate (15 g), IPDI (7 g) and TEOS (2 g) were mixed and stirred at $30^{\circ} \mathrm{C}$ for $15 \mathrm{~min}$ to form a homogeneous oil phase. Then, the oil phase was mixed with aqueous phase by a high-shear dispersion homogenizer at the speed of $5000 \mathrm{rpm}$ for $10 \mathrm{~min}$ to form stable emulsion in a beaker. After that, DETA $(7 \mathrm{~g})$ was slowly dropped into the emulsion at a 
stirring rate of $500 \mathrm{rpm}$ for $2 \mathrm{~h}$ at $40{ }^{\circ} \mathrm{C}$. Then, the temperature was raised to $70{ }^{\circ} \mathrm{C}$ rapidly, and then, the emulsion was cured under $500 \mathrm{rpm}$ of agitation for $2 \mathrm{~h}$ to form polyurethane-SiO $\mathrm{S}_{2}$ composite microcapsule slurry.

\subsubsection{Preparation of Polyurea-Polyurethane- $\mathrm{SiO}_{2}$ Microcapsules}

The microcapsules were synthesized by the interfacial polymerization. Two different phases were prepared, PEG-400 (3 g) and SMA (5 g) were dissolved into deionized water, and formed the aqueous phase. Ethyl palmitate (15 g), IPDI $(7 \mathrm{~g})$, and TEOS $(2 \mathrm{~g})$ were mixed and stirred at $30{ }^{\circ} \mathrm{C}$ for $15 \mathrm{~min}$ to form a homogeneous oil phase, then the oil phase was mixed with aqueous phase by a high-shear dispersion homogenizer at the speed of $5000 \mathrm{rpm}$ for $10 \mathrm{~min}$ to form an emulsion in a beaker. After, DETA (4 g) was slowly dropped into the emulsion at a stirring rate of $500 \mathrm{rpm}$ for $2 \mathrm{~h}$ with a mechanical agitation, while the temperature of the solution was controlled at $40^{\circ} \mathrm{C}$, then the temperature was raised to $70{ }^{\circ} \mathrm{C}$ rapidly, and the emulsion was cured under $500 \mathrm{rpm}$ of agitation for $2 \mathrm{~h}$ to form a polyurea-polyurethane- $\mathrm{SiO}_{2}$ MicroPCMs slurry. After being washed by hot distilled water, the prepared MicroPCMs were then treated by filtration and drying.

\subsection{Characterization}

\subsubsection{Fourier Transform Infrared Spectroscopy (FT-IR)}

FT-IR spectrum, recorded on a FT-IR Uecior 22 spectrophotometer (Shanghai, China), was used to identify the structure of the microcapsules. Samples were prepared as $\mathrm{KBr}$ pellets, and scanned against a blank KBr pellet background at wave numbers ranging from 4000 to $500 \mathrm{~cm}^{-1}$ with a resolution of $4.0 \mathrm{~cm}^{-1}$.

\subsubsection{Field Emission-Scanning Electron Microscopy (FE-SEM)}

A scanning electron microscope (SEM, Hitachi S-4800, Tokyo, Japan) was used to observe the morphology and structures of these composite microcapsules. The samples were prepared by dropping an aliquot $(20 \mu \mathrm{L})$ of microcapsules onto a slide; subsequently, the slide was mounted on metal stubs using conductive tape and vacuum-coated with a thin layer of platinum using a sputter coater.

\subsubsection{The Phase Change Properties Analysis}

The phase change properties were characterized using a differential scanning calorimeter (DSC, NETZSCH $200 \mathrm{~F} 3$, Bavaria, Germany) in the range of $-20-80{ }^{\circ} \mathrm{C}$. A specimen of $5 \sim 10 \mathrm{mg}$ was encapsulated in an aluminum pan under a nitrogen atmosphere and first heated from -20 to $80^{\circ} \mathrm{C}$ at a rate of $10^{\circ} \mathrm{C} / \mathrm{min}$ and kept at $80^{\circ} \mathrm{C}$ for $2 \mathrm{~min}$. Subsequently, the specimen was cooled to $-30^{\circ} \mathrm{C}$ at a rate $10^{\circ} \mathrm{C} / \mathrm{min}$ and kept at that temperature for $2 \mathrm{~min}$. Finally, the specimen was heated again from -20 to $80{ }^{\circ} \mathrm{C}$ with a rate of $10{ }^{\circ} \mathrm{C} / \mathrm{min}$. DSC thermograms in the first cooling and the second heating process were recorded.

\subsubsection{Thermal Gravimetric Analysis (TGA)}

The thermal stabilities of the samples were investigated using the thermogravimetric analyzer (TGA, Netzsch, STA409PC, Bavaria, Germany) in the range of $40-550{ }^{\circ} \mathrm{C}$ with a heating rate of $10^{\circ} \mathrm{C} / \mathrm{min}$ under a nitrogen atmosphere.

\subsection{Compactness Test}

The prepared microcapsules $(10.00 \mathrm{~g})$ were packed in conical filter membrane and stored at $120^{\circ} \mathrm{C}$ for $1 \mathrm{~h}$. Quality of microcapsules is measured every ten minutes, and then the change of the weight loss rates could be examined. 


\section{Results and Discussion}

\subsection{Morphology and Inner Microstructure of Various MicroPCMs}

Figure 1 presented the effects of various core/shell ratios on morphology of polyurethane MicroPCMs. During SEM sample preparation, we used a glass plate to apply certain force onto the capsules after they were adhered onto the conductive carbon tape. When the core/shell ratio was 1:1 (a), the spherical microcapsules were integrated, and the smooth surfaces were compact, however, there were some dents on the surface. When the ratio increased to 2:1 (b), few cracking phenomena appeared on the surface of capsule, and more cracking phenomena appeared when core/shell ratio enhanced to 3:1. The exact value of shell strength cannot be obtained, however, the strength of various capsules could be simply compared. The strength mechanical performance and stability decreased with shell thickness turned thicker and thicker accordingly.

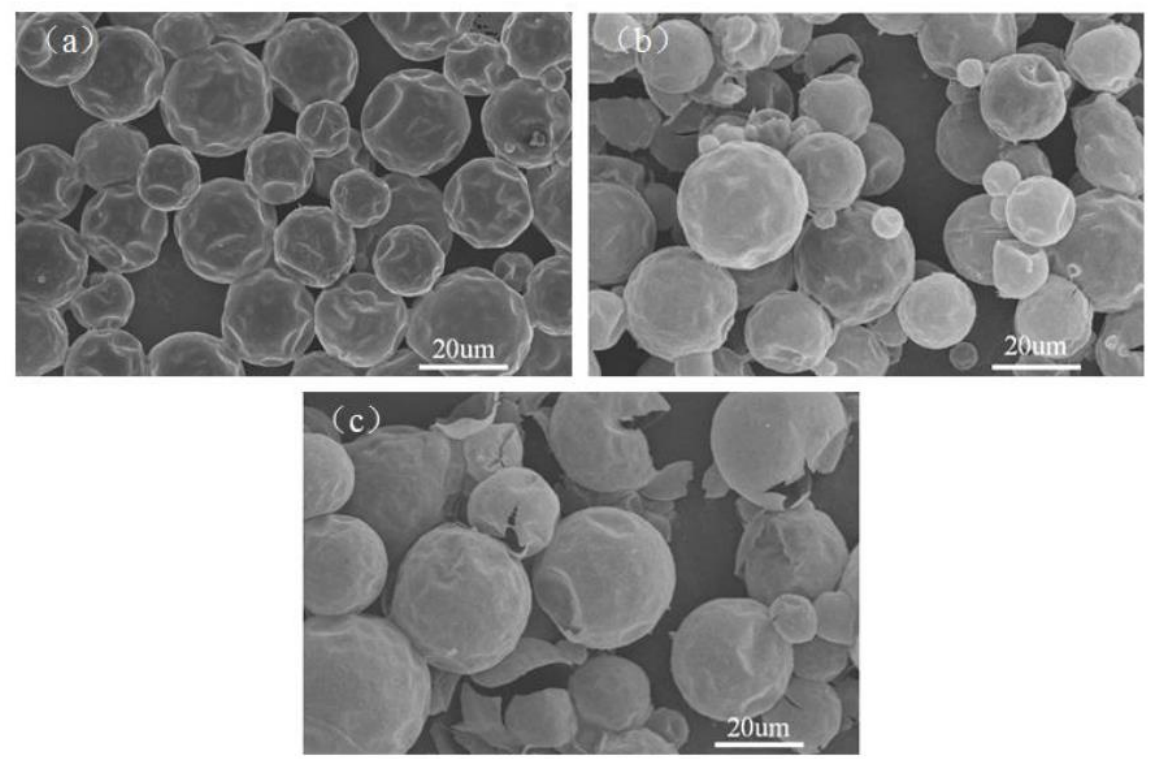

Figure 1. Effects of various core/shell ratios on morphology of polyurethane MicroPCMs: (a) 1:1; (b) 2:1; and (c) 3:1.

SEM images of all MicroPCMs were presented in Figure 2. The shape of the polyurea microcapsules was spherical, and the surface was seriously dented. While the polyurethane microcapsules were regular spherical with relatively smooth surfaces, the dents have been greatly improved, and there was only a small degree of denting. The reason was probably due partly to the fact that the stiffness and mechanical performance of polyurethane shell synthesized between IPDI and DETA was enhanced, compared with that of polyurea shell containing PEG soft segments, which is prone to depress or deform when the microcapsule is subjected to external forces or collision of particles. By contrast, the polyurethane shell composed of high crosslinked amide bonds possessed high strength mechanical performance, and could withstand the outer force. Compared with polyurea microcapsules, the surface depression of hybrid polyurea- $\mathrm{SiO}_{2}$ microcapsules have turned out slightly better, this was mainly because the polyurea shell material was formed already, before the $\mathrm{SiO}_{2}$ protective layer was formed inside, caused by relatively slower hydrolysis polymerization. The protective inner $\mathrm{SiO}_{2}$ layer almost had no influence on the surface of polyurea shell, but played a certain supporting role. The shape of polyurethane- $\mathrm{SiO}_{2}$ microcapsules were more spherical and the surfaces are smooth. When the TEOS hydrolysis occurred, the polymerization shrinkage forced on polyurethane shell increased, and resulted in the dent appearance on the polyurethane- $\mathrm{SiO}_{2}$ shell. 

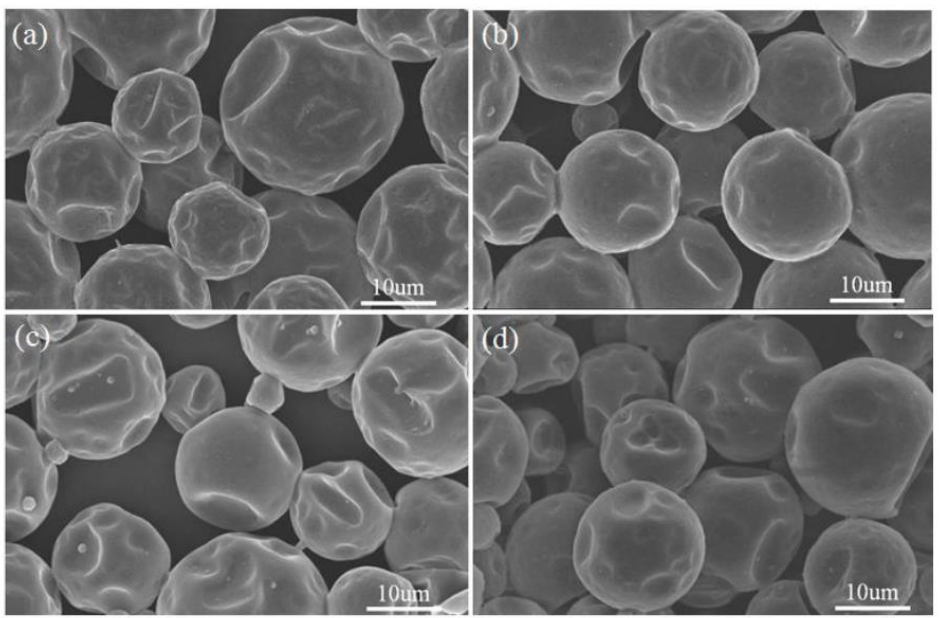

Figure 2. SEM micrographs of various MicroPCMs: (a) polyurea microcapsules; (b) polyurethane microcapsules; (c) polyurea- $\mathrm{SiO}_{2}$ microcapsules; (d) polyurethane- $\mathrm{SiO}_{2}$ microcapsules.

As presented in Figure 3, it can be seen from the inner shell surfaces of various microcapsules that the inner surface of the polyurea microcapsules was rough, as shown in Figure 3a. Relatively speaking, the inner surface of the polyurethane microcapsules became much more smooth in Figure $3 \mathrm{~b}$. Many hemispherical convex parts appeared on the inner shell of both (c) polyurea-SiO${ }_{2}$ microcapsules and (d) polyurethane- $\mathrm{SiO}_{2}$ microcapsules, which were possibly a result of hydrolysis polymerization of TEOS. The bulging of the inner surface indicated that the silica formed was composed with the polyurethane from the tetraethoxysilane.
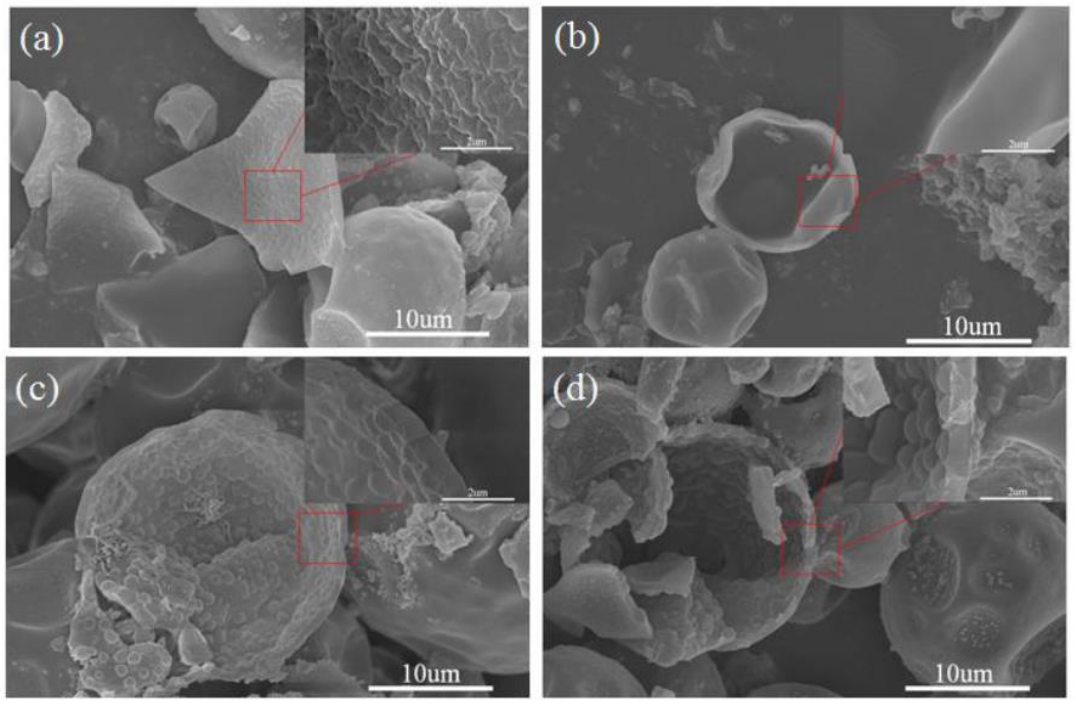

Figure 3. SEM micrographs of inner shell of various MicroPCMs: (a) polyurea microcapsules;

(b) polyurethane microcapsules; (c) polyurea- $\mathrm{SiO}_{2}$ microcapsules; (d) polyurethane- $\mathrm{SiO}_{2}$ microcapsules.

Figure 4 showed the influence of various addition amounts of TEOS on morphology of polyurethane MicroPCMs. The spherical microcapsules with smooth and compact surface had integrity without TEOS addition. With the increase of addition amounts of TEOS, the degree of concave on the shell became a little more significant, this phenomenon may be attributed to that some TEOS did not polymerized by hydrolysis completely, or the resultant $\mathrm{SiO}_{2}$ products did not form a continuous enhanced layer inside the shell. 

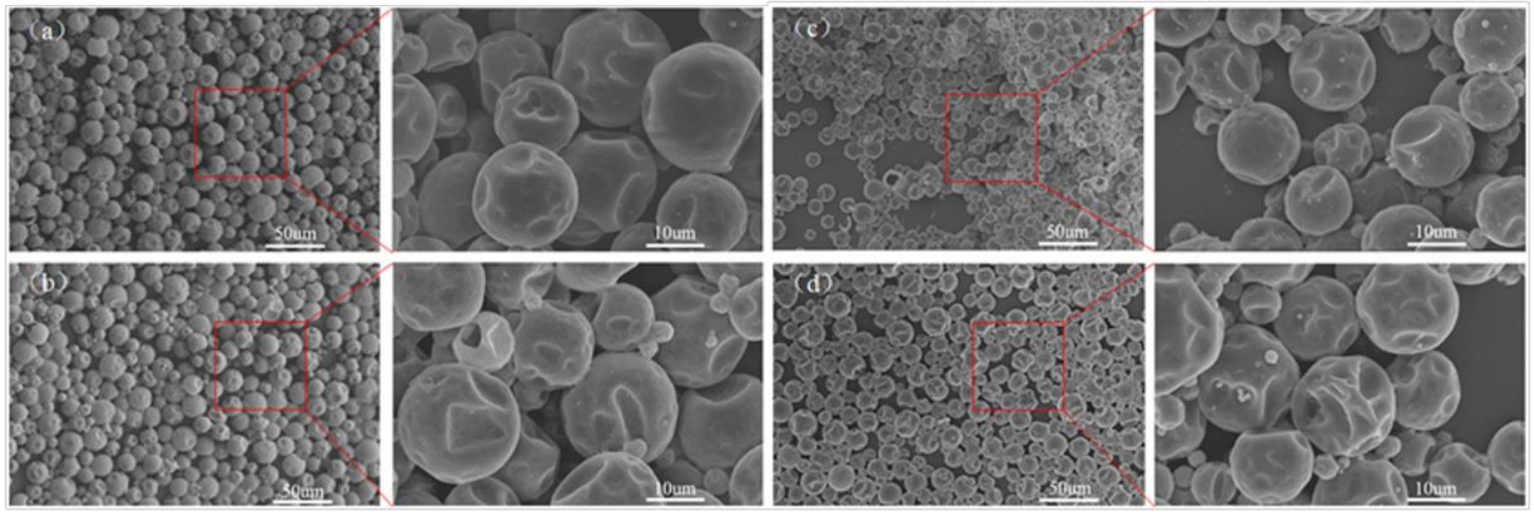

Figure 4. Influence of various addition amounts of TEOS on morphology of polyurethane MicroPCMs: (a) $0 \mathrm{~g}$; (b) $2 \mathrm{~g}$; (c) $4 \mathrm{~g}$; and (d) $6 \mathrm{~g}$.

\subsection{FT-IR Spectroscopy Analysis of Microcapsules}

The FT-IR spectra of four kind MicroPCMs were preseted and compared in Figure 5. An absorption band at $2256 \mathrm{~cm}^{-1}$ belonged to the isocyanate group (-NCO) in spectra (e) (Figure 5). As monomer IPDI was polymerized to form polyurethane or polyurea polymer, the absorption band at $2256 \mathrm{~cm}^{-1}$ disappeared in spectra (a), (b), (c), and (d) (Figure 5), indicating that there was no isocyanate residue left in the microcapsules shell. There were several reasons for this phenomenon. Firstly, the isocyanate group of the IPDI reacted fast with the amine group of the DETA. Secondly, DETA was compatible with the ethyl palmitate, so it could diffuse into the interface of the emulsion and ensured the-NCO in IPDI was completely reacted. The peaks at 2925 and $2845 \mathrm{~cm}^{-1}$ in spectra (a) were respectively assigned to methylene and methyne $\mathrm{C}-\mathrm{H}$ in the ethyl palmitategroup. The peak at $1735 \mathrm{~cm}^{-1}$ in spectrum (a) was assigned to the $\mathrm{C}=\mathrm{O}$ in ethyl palmitate group. The peaks at $1250 \mathrm{~cm}^{-1}$ in spectra $(\mathrm{b})$ were assigned to $\mathrm{C}-\mathrm{O}-\mathrm{C}$ in the polyurethane polymer chain. The results indicated that the polyurethane were probably polymerized. The FT-IR spectra of MicroPCMs (c) and (d) containing $\mathrm{SiO}_{2}$ were compared in Figure 5. Both of the spectra (c) and (d) possessed almost identical absorption band at $1084 \mathrm{~cm}^{-1}$, which were respectively assigned to $\mathrm{C}-\mathrm{O}-\mathrm{C}$. These results indicated that the $\mathrm{SiO}_{2}$ had been polymerized through TEOS hydrolysis and polymerization reaction.

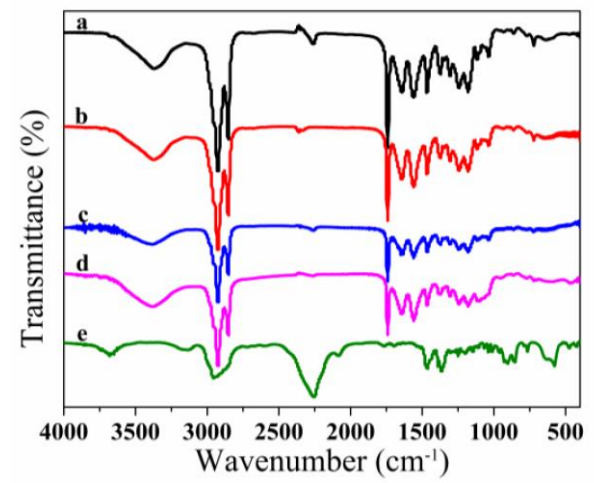

Figure 5. FT-IR spectra of various MicroPCMs and shell-forming monomer: (a) polyurea microcapsules; (b) polyurethane microcapsules; (c) polyurea- $\mathrm{SiO}_{2}$ microcapsules; (d) polyurethane- $\mathrm{SiO}_{2}$ microcapsules; (e) IPDI monomer.

\subsection{EDS Energy Spectra Analysis}

From Figure $6 \mathrm{a}$, it can be found the element $\mathrm{C}, \mathrm{N}$, and $\mathrm{O}$ of polyurea microcapsules were 64.40 , 20.71 and $14.89 \mathrm{wt} \%$, respectively. Accordingly, the element C, N, and O of polyurethane microcapsule 
were $69.19,14.16$, and $16.66 \mathrm{wt} \%$, respectively. Figure $7 \mathrm{a}, \mathrm{a}_{1}, \mathrm{~b}, \mathrm{~b}_{1}$ showed the EDS data of polyurea-SiO${ }_{2}$ microcapsules and polyurethane- $-\mathrm{SiO}_{2}$ microcapsules. The polyurea- $\mathrm{SiO}_{2}$ or polyurethane- $\mathrm{SiO}_{2}$ MicroPCMs composites were consisted of ethyl palmitate cores, $\mathrm{SiO}_{2}$, and polyurea or polyurethane, which contained silicon $(\mathrm{Si})$, carbon $(\mathrm{C})$, oxygen $(\mathrm{O})$, hydrogen $(\mathrm{H})$, and nitrogen $(\mathrm{N})$. The silicon (Si) weight contents of outer and inner shell of polyurea- $\mathrm{SiO}_{2}$ capsule were measured as $2.00 \mathrm{wt} \%$ and $10.13 \mathrm{wt} \%$, respectively. While the silicon $(\mathrm{Si})$ weight contents of outer and inner shell of polyurethane- $\mathrm{SiO}_{2}$ composite capsule were $3.31 \mathrm{wt} \%$ and $7.13 \mathrm{wt} \%$, respectively. As can be seen from the EDS spectra of the inner and outer surfaces of both polyurea-SiO${ }_{2}$ microcapsules and polyurethane- $\mathrm{SiO}_{2}$ microcapsules, the content of silicon contained on the inner surface was obviously higher than that of the silicon contained on the outer surface, which further illustrated that the hydrolysis polymerization mainly occurred inside the shell rather than other position of shell. The $\mathrm{Si}$ characteristic peaks and corresponding data indicated that the $\mathrm{SiO}_{2}$ was possibly formed on the inner shell by the hydrolysis of TEOS, and the microscale semisphere Si composites could also be clearly seen from the Figure $7 a_{1}, b_{1}$.

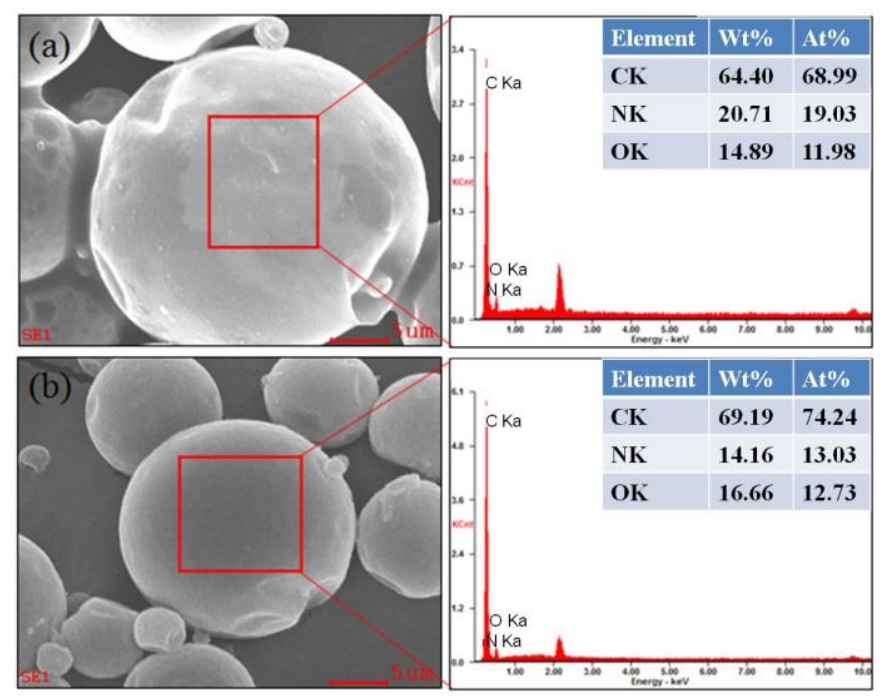

Figure 6. The EDS of MicroPCMs: (a) polyurea microcapsules; (b) polyurethane microcapsules.
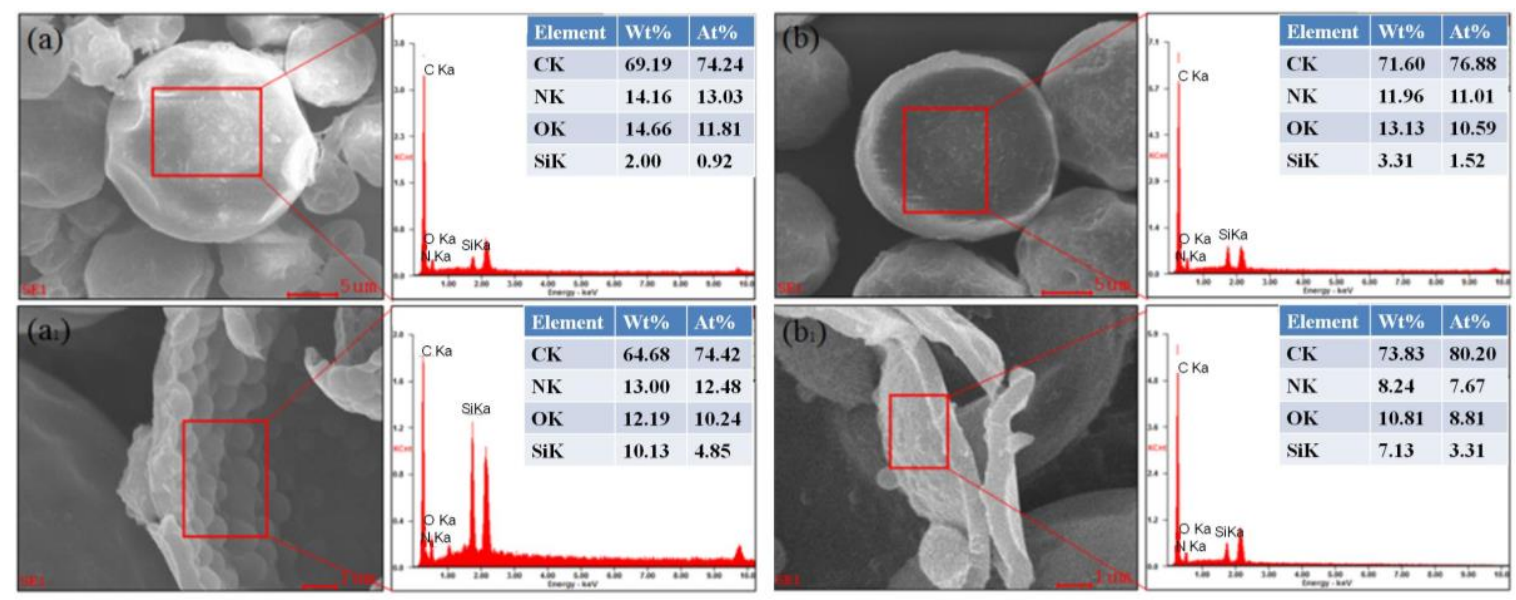

Figure 7. The EDS of (a) and $\left(\mathbf{a}_{1}\right)$ polyurea-SiO${ }_{2}$ microcapsules; $(\mathbf{b})$ and $\left(\mathbf{b}_{1}\right)$ polyurethane$\mathrm{SiO}_{2}$ microcapsules. 


\subsection{Compactness Analysis}

The weight loss rates of various MicroPCMs were shown and compared in Figure 8 . The weight of the microcapsules decreased with increasing heat-treatment time in Figure 8. The weight loss rate of polyurea microcapsules was approximately $11.5 \%$, while for polyurethane microcapsules, it was about $9.9 \%$. Compared with polyurea microcapsules, the compactness of polyurethane microcapsules were little enhanced, indicating that there was some effect on improving the compactness of MicroPCMs with reduced denting. By contrast, the weight loss rates of polyurea- $\mathrm{SiO}_{2}$ microcapsules and polyurethane- $\mathrm{SiO}_{2}$ microcapsules were around $8.0 \%$ and $7.9 \%$, respectively. The mass loss rate of both polyurea- $\mathrm{SiO}_{2}$ and polyurethane- $\mathrm{SiO}_{2}$ microcapsules was significantly reduced, indicating that $\mathrm{SiO}_{2}$ composition shell formed by the hydrolysis polymerization of TEOS, deceased the porosity of microcapsules and enhanced the compactness of microcapsules.

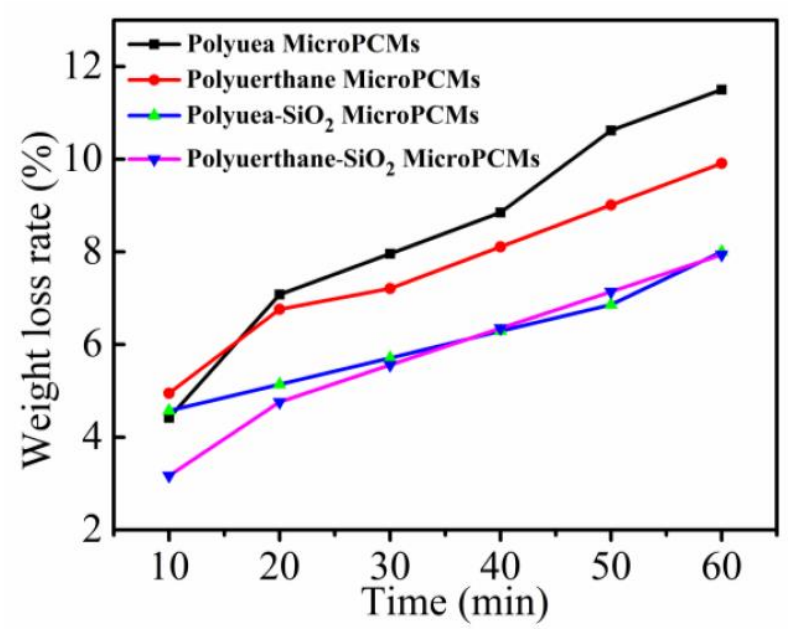

Figure 8. Weight loss rates curve comparison of various MicroPCMs.

\subsection{Melting and Crystallization Behaviors of MicroPCMs}

The DSC curves of various MicroPCMs and pure ethylpalmitate were shown in Figure 9. Various MicroPCMs had single endothermic peak at range of $25.5-31.6^{\circ} \mathrm{C}$. While two peaks from high to low temperature were observed on the DSC cooling curve of pure ethyl palmitate attributed to the formation of different crystal form or transformation of crystal type, and the latent heat was $203 \mathrm{~J} / \mathrm{g}$ approximately. Interestingly, sole peak from high to low temperature could be observed on the DSC cooling curve of MicroPCMs. The reason was that the shell of MicroPCMs had a microscale space restriction effect and heat insulation effect on ethyl palmitate core. The encapsulation ratios could be calculated using

$$
\eta \%=\frac{\Delta H_{\text {MicroPCMs }}}{\Delta H \times \frac{M_{\mathrm{c}}}{M_{\text {MicroPCMs }}}} \times 100
$$

where $\Delta H_{\text {MicroPCMs }}$ represented the melting enthalpy of MicroPCMs, and $\Delta H_{\mathrm{c}}(203 \mathrm{~J} / \mathrm{g})$ represented the melting enthalpy of ethyl palmitate as measured by the DSC. $M_{\text {MicroPCMs }}$ and $M_{\mathrm{c}}$ were the mass of the microcapsules raw materials and bulk ethyl palmitate, respectively. The encapsulation ratio of all MicroPCMs exceed in 100\%, by calculation, indicating that the shell contents of MicroPCMs were less than theoretical values. There was a few main reasons accounting for that the polymerization reaction of shell between shell-forming monomers, such as IPDI, DETA or PEG 400, could not react completely. Moreover, the phase change enthalpies on exothermic peak and endothermic peak of polyurethane- $\mathrm{SiO}_{2}$ microcapsules are $126.1 \mathrm{~J} . \mathrm{g}^{-1}$ and $122.1 \mathrm{~J} . \mathrm{g}^{-1}$, and the PCM content of MicroPCMs can be calculated by the ratio of ethyl palmitate of MicroPCMs to that of corresponding ethyl palmitate bulk. The average enthalpy of polyurethane- $\mathrm{SiO}_{2}$ microcapsules is as high as $124.1 \mathrm{~J} . \mathrm{g}^{-1}$, and the 
measured PCM content can be calculated as $61.0 \mathrm{wt} \%$. Observing the DSC cooling curve of MicroPCMs, the supercooling appears in all MicroPCMs samples (Table 1) to some extent. The supercooling was probably caused by decreasing nucleating agents in microscopic space and confined crystallizaion, and the crystallization temperature reduced with the decrease of particle diameter. Due to the reducing crystallization temperature, the latent heat was released at a lower temperature and in a wider temperature range.

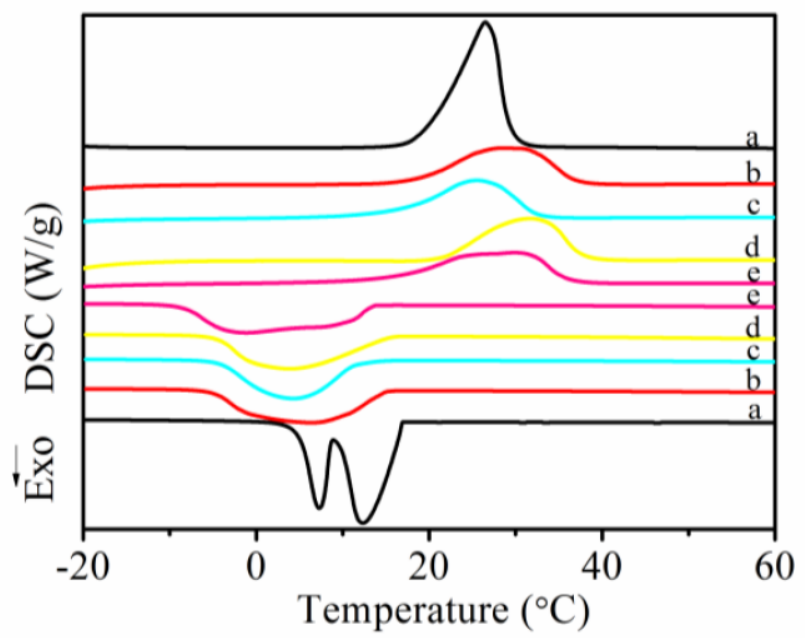

Figure 9. DSC thermograms comparison of various MicroPCMs and pure ethyl palmitate: (a) ethyl palmitate; (b) polyurea MicroPCMs; (c) polyurethane MicroPCMs; (d) polyurea-SiO $2 \mathrm{MicroPCMs}^{-}$ (e) polyurethane- $\mathrm{SiO}_{2}$ MicroPCMs.

Table 1. Phase change performance comparison of various MicroPCMs and pure ethyl palmitate.

\begin{tabular}{|c|c|c|c|c|c|c|c|c|c|c|}
\hline Sample & $\begin{array}{l}T_{\text {mo }}{ }^{a} \\
\left({ }^{\circ} \mathrm{C}\right)\end{array}$ & $\begin{array}{l}T_{\mathrm{mp}}{ }^{b} \\
\left({ }^{\circ} \mathrm{C}\right)\end{array}$ & $\begin{array}{l}T_{\mathrm{me}}{ }^{\mathrm{c}} \\
\left({ }^{\circ} \mathrm{C}\right)\end{array}$ & $\begin{array}{l}\Delta H_{\mathrm{m}} \\
\left(\mathrm{J} \cdot \mathrm{g}^{-1}\right)\end{array}$ & $\begin{array}{l}T_{\text {co }}{ }^{d} \\
\left({ }^{\circ} \mathrm{C}\right)\end{array}$ & $\begin{array}{l}T_{\mathrm{cp}} \text { e } \\
\left({ }^{\circ} \mathrm{C}\right)\end{array}$ & $\begin{array}{l}T_{\mathrm{ce}} \mathrm{f} \\
\left({ }^{\circ} \mathrm{C}\right)\end{array}$ & $\begin{array}{l}\Delta H_{\mathrm{c}} \\
\left(\mathrm{J} \cdot \mathrm{g}^{-1}\right)\end{array}$ & $\begin{array}{l}\Delta H_{\mathrm{i}} \mathrm{g} \\
\left(\mathrm{J} \cdot \mathrm{g}^{-1}\right)\end{array}$ & $\begin{array}{l}\text { PCM content } \\
\text { (wt } \%)\end{array}$ \\
\hline a & 19.6 & 26.5 & 29.3 & 203.9 & 16.9 & 12.3 & 8.8 & 202.7 & 203.3 & - \\
\hline$b$ & 18.7 & 28.5 & 36.9 & 123.4 & 15.0 & 6.2 & -5.0 & 128.6 & 126.0 & 62.0 \\
\hline c & 16.9 & 25.5 & 32.4 & 110.5 & 11.5 & 4.2 & -4.1 & 113.7 & 112.1 & 55.1 \\
\hline d & 21.9 & 31.6 & 37.5 & 120.1 & 15.3 & 3.7 & -4.4 & 121.0 & 120.5 & 59.3 \\
\hline e & 16.5 & 29.8 & 35.8 & 122.1 & 13.3 & -1.2 & -8.3 & 126.1 & 124.1 & 61.0 \\
\hline
\end{tabular}

(a) ethyl palmitate; (b) polyurea microcapsules; (c) polyurethane microcapsules; (d) polyurea- $\mathrm{SiO}_{2}$ microcapsules; (e) polyurethane- $\mathrm{SiO}_{2}$ microcapsules. Note: ${ }^{\mathrm{a}}$ onset temperature on DSC heating curve; ${ }^{\mathrm{b}}$ peak temperature on DSC heating curve; ${ }^{c}$ enthalpy on DSC heating curve; ${ }^{\mathrm{d}}$ onset temperature on DSC cooling curve; ${ }^{\mathrm{e}}$ peak temperature on DSC cooling curve; ${ }^{\mathrm{f}}$ enthalpy on DSC cooling curve; ${ }^{\mathrm{g}}$ average enthalpy of $\left|\Delta H_{\mathrm{m}}\right|$ and $\mid \Delta H_{\mathrm{c}} \mathrm{l}$; ${ }^{\mathrm{h}}$ ratio of $\Delta H$ of MicroPCMs to that of corresponding ethyl palmitate bulk.

\subsection{Thermal Stability of MicroPCMs}

Thermal stability was considered as an important performance of encapsulated PCMs for their practical application. The thermal stabilities of ethyl palmitate, polyurea MicroPCMs, polyurethane MicroPCMs, polyurea- $\mathrm{SiO}_{2}$ and polyurethane- $\mathrm{SiO}_{2}$ MicroPCMs were evaluated by means of TGA, as shown in Figure 10. The weight of ethyl palmitate, polyurea MicroPCMs, polyurethane MicroPCMs, polyurea- $-\mathrm{SiO}_{2}$ and polyurethane- $\mathrm{SiO}_{2}$ MicroPCMs decreased with increasing temperature. Pure ethyl palmitate began to evaporate and lose weight at approximately $195.1^{\circ} \mathrm{C}$, and lost weight completely at approximately $236.2^{\circ} \mathrm{C}$. The thermal resistant temperature of polyurea MicroPCMs was about $217.7^{\circ} \mathrm{C}$, indicating that the ethyl palmitate encapsulated in shells probably decomposed, and evaporated at a higher temperature, owing to the protection of the polyurea microcapsule shell. The thermal resistant temperature of polyurethane MicroPCMs was around $215.6{ }^{\circ} \mathrm{C}$, which was similar with that of polyurea MicroPCMs. While the thermal resistant temperature of MicroPCMs with $\mathrm{SiO}_{2}$-polyurea composite shell was $213.4^{\circ} \mathrm{C}$, there was no enhancement in thermal stability, indicating that there was no effect on 
the compactness and thermal stability of shell by incorporation of $\mathrm{SiO}_{2}$. However, the thermal stability of MicroPCMs with polyurethane- $\mathrm{SiO}_{2}$ composite shell was slightly increased to around $233.0^{\circ} \mathrm{C}$ from $215.6{ }^{\circ} \mathrm{C}$ of polyurethane MicroPCMs, indicating more compact and stable organic-inorganic microstructure was likely formed in the polyurethane- $\mathrm{SiO}_{2}$ composite shell.

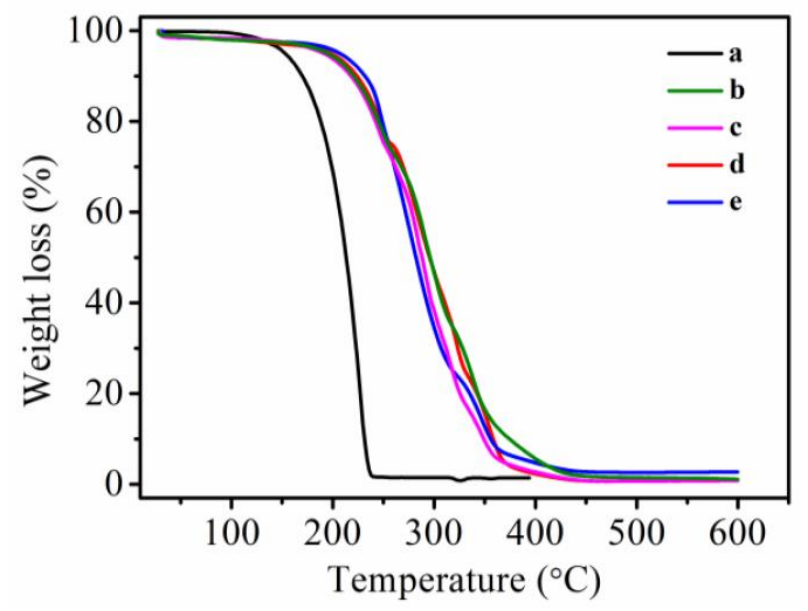

Figure 10. Thermal gravity analysis (TGA) curve comparison of various MicroPCMs and pure ethyl palmitate: (a) ethyl palmitate; (b) polyurea microcapsules; (c) polyurethane microcapsules; (d) polyurea- $\mathrm{SiO}_{2}$ microcapsules; (e) polyurethane- $\mathrm{SiO}_{2}$ microcapsules.

\section{Conclusions}

In the current study, we synthesized stable phase change microcapsule by emulsion polymerization and interfacial polymerization method. FE-SEM micrographs showed the optimal core/shell ratios of polyurethane MicroPCMs was 1:1, and the suitable addition of TEOS was $2 \mathrm{~g}$. The smoothness and compactness of both polyurea- $\mathrm{SiO}_{2}$ and polyurethane- $\mathrm{SiO}_{2}$ microcapsules enhanced slightly when compared with that without TEOS. The melting enthalpy of polyurea- $\mathrm{SiO}_{2}$ microcapsules and polyurethane- $\mathrm{SiO}_{2}$ were 120.1 and $121.1 \mathrm{~J} \cdot \mathrm{g}^{-1}$, while the crystallization enthalpy of polyurea- $\mathrm{SiO}_{2}$ microcapsules and polyurethane- $\mathrm{SiO}_{2}$ were 122.1 and $126.1 \mathrm{~J} \cdot \mathrm{g}^{-1}$, respectively. The $\mathrm{SiO}_{2}$ composite shell had good effect on thermal compactness, and the weight loss rate of polyurea- $\mathrm{SiO}_{2}$ microcapsules and polyurethane- $\mathrm{SiO}_{2}$ microcapsules decreased $3.5 \%$ and $4.1 \%$, respectively. The TGA results also showed that polyurethane- $\mathrm{SiO}_{2}$ microcapsules decreased $16{ }^{\circ} \mathrm{C}$, indicating composites shell could improve thermal stability of the polyurea microcapsules.

Author Contributions: Conceptualization, W.L.; Methodology, Z.Z. and W.L.; Software, M.G.; Validation, M.G.; Formal Analysis, Q.Y.; Investigation, Y.W.; Resources, J.W.; Data Curation, Q.Y.; Writing-Original Draft Preparation, Z.Z.; Writing-Review \& Editing, J.W.; Visualization, Y.W.; Supervision, X.Z.; Project Administration, X.Z.; Funding Acquisition, W.L.

Funding: This research was funded by National Natural Science Foundation of China (No. 51573135 and No. 51203113), Tianjin Research Program of Application Foundation and Advanced Technology (No. 16JCYBJC17100) and The Science and Technology Plans of Tianjin (No. 15PTSYJC00230).

Conflicts of Interest: The authors declare no conflict of interest.

\section{References}

1. Anghel, E.M.; Georgiev, A.; Petrescu, S.; Popov, R.; Constantinescu, M. Thermo-physical characterization of some paraffins used as phase change materials for thermal energy storage. J. Therm. Anal. Calorim. 2014, 117, 557-566. [CrossRef]

2. Milian, Y.E.; Gutierrez, A.; Grageda, M.; Ushak, S. A review on encapsulation techniques for inorganic phase change materials and the influence on their thermophysical properties. Renew. Sustain. Energy Rev. 2017, 73, 983-999. [CrossRef] 
3. Chang, S.J.; Wi, S.; Jeong, S.G.; Kim, S. Analysis on phase transition range of the pure and mixed phase change materials (PCM) using a thermostatic chamber test and differentiation. J. Therm. Anal. Calorim. 2017, 131, 1999-2004. [CrossRef]

4. Motahar, S.; Alemrajabi, A.A.; Khodabandeh, R. Experimental investigation on heat transfer characteristics during melting of a phase change material with dispersed $\mathrm{TiO}_{2}$ nanoparticles in a rectangular enclosure. Int. J. Heat Mass Transf. 2017, 109, 134-146.

5. Geng, X.Y.; Li, W.; Wang, Y.; Lu, X.W.; Wang, J.P.; Wang, N.; Li, J.J.; Zhang, X.X. Reversible thermochromic microencapsulated phase change materials for thermal energy storage application in thermal protective clothing. Appl. Energy 2018, 217, 281-294. [CrossRef]

6. Raghunanan, L.; Floros, M.C.; Narine, S.S. Thermal stability of renewable diesters as phase change materials. Thermochim. Acta 2016, 644, 61-68. [CrossRef]

7. Guo, M.; Li, W.; Han, N.; Wang, J.; Su, J.; Li, J.; Zhang, X. Novel dual-component microencapsulated hydrophobic amine and microencapsulated isocyanate used for self-healing anti-corrosion coating. Polymers 2018, 10, 319. [CrossRef]

8. Zhang, H.; Shi, Y.; Shentu, B.; Weng, Z. Synthesis and thermal performance of polyurea microcapsulated phase change materials by interfacial polymerization. Polym. Sci. Ser. B 2017, 59, 689-696. [CrossRef]

9. Fredi, G.; Dorigato, A.; Fambri, L.; Pegoretti, A. Wax confinement with carbon nanotubes for phase changing epoxy blends. Polymers 2017, 9, 405. [CrossRef]

10. Fredi, G.; Dorigato, A.; Fambri, L.; Pegoretti, A. Multifunctional epoxy/carbon fiber laminates for thermal energy storage and release. Compos. Sci. Technol. 2018, 158, 101-111. [CrossRef]

11. Li, M.; Liu, J.P.; Shi, J.B. Synthesis and properties of phase change microcapsule with $\mathrm{SiO}_{2}-\mathrm{TiO}_{2}$ hybrid shell. Sol. Energy 2018, 167, 158-164. [CrossRef]

12. Lian, Q.; Li, Y.; Sayyed, A.A.S.; Cheng, J.; Zhang, J.Y. Facile strategy in designing epoxy/paraffin multiple phase change materials for thermal energy storage applications. ACS Sustain. Chem. Eng. 2018, 6, 3375-3384. [CrossRef]

13. Esapour, M.; Hosseini, M.J.; Ranjbar, A.A.; Pahamli, Y.; Bahrampoury, R. Phase change in multi-tube heat exchangers. Renew. Energy 2016, 85, 1017-1025. [CrossRef]

14. Michaelides, E.E. A new model for the lifetime of fossil fuel resources. Nat. Resour. Res. 2016, 26, 161-175. [CrossRef]

15. Khan, Z.; Khan, Z.; Ghafoor, A. A review of performance enhancement of PCM based latent heat storage system within the context of materials, thermal stability and compatibility. Energy Convers. Manag. 2016, 115, 132-158. [CrossRef]

16. Abdollahzadeh, M.; Esmaeilpour, M. Enhancement of phase change material (PCM) based latent heat storage system with nano fluid and wavy surface. Int. J. Heat Mass Transf. 2015, 80, 376-385. [CrossRef]

17. Pasupathy, A.; Velraj, R.; Seeniraj, R.V. Phase change material-based building architecture for thermal management in residential and commercial establishments. Renew. Sustain. Energy Rev. 2008, 12, 39-64. [CrossRef]

18. Goeke, J.; Henne, A. Time-temperature charge function of a high dynamic thermal heat storage with phase change material. Energy Power Eng. 2015, 7, 41-54. [CrossRef]

19. Luo, J.; Zhao, L.; Yang, Y.; Song, G.; Liu, Y.; Chen, L.; Tang, G. Emulsifying ability and cross-linking of silk fibroin microcapsules containing phase change materials. Sol. Energy Mater. Sol. Cells 2016, 147, 144-149. [CrossRef]

20. Sun, N.; Xiao, Z. Paraffin wax-based phase change microencapsulation embedded with silicon nitride nanoparticles for thermal energy storage. J. Mater. Sci. 2016, 51, 8550-8561. [CrossRef]

21. Wei, H.; Zhao, Z.; Wei, C.; Yu, G.; Liu, Z.; Zhang, B.; Bian, J.; Bian, Z.; Huang, C. Antiphotobleaching: A type of structurally rigid chromophore ready for constructing highly luminescent and highly photostable europium complexes. Adv. Funct. Mater. 2016, 26, 2085-2096. [CrossRef]

22. Tillet, G.; Boutevin, B.; Ameduri, B. Chemical reactions of polymer crosslinking and post-crosslinking at room and medium temperature. Prog. Polym. Sci. 2011, 36, 191-217. [CrossRef]

23. Zhu, Y.; Qin, Y.; Wei, C.; Liang, S.; Luo, X.; Wang, J.; Zhang, L. Nanoencapsulated phase change materials with polymer-SiO 2 hybrid shell materials: Compositions, morphologies, and properties. Energy Convers. Manag. 2018, 164, 83-92. [CrossRef] 
24. Lee, S.M.; Park, J.Y.; Park, S.B. Comparative study of the storage stability between a melamine-ureaformaldehyde and a urea-formaldehyde resin. For. Prod. J. 2012, 62, 146-149. [CrossRef]

25. Despres, A.; Pizzi, A. Colloidal aggregation of aminoplasticpolycondensation resins: Urea-formaldehyde versus melamine-formaldehyde and melamine-urea-formaldehyde resins. J. Appl. Polym. Sci. 2006, 100, 1406-1412. [CrossRef]

26. Calle, M.; Lligadas, G.; Ronda, J.C.; Galia, M.; Cadiz, V. Non-isocyanate route to biobased polyurethanes and polyureas via AB-type self-polycondensation. Eur. Polym. J. 2016, 84, 837-848. [CrossRef]

27. Zhang, F.; Jiang, X.; Zhu, X.; Chen, Z.; Kong, X.Z. Preparation of uniform and porous polyurea microspheres of large size through interfacial polymerization of toluene diisocyanate in water solution of ethylene diamine. Chem. Eng. J. 2016, 303, 48-55. [CrossRef]

28. Tatiya, P.D.; Hedaoo, R.K.; Mahulikar, P.P.; Gite, V.V. Novel polyurea microcapsules using dendritic functional monomer: Synthesis, characterization, and its use in self-healing and anticorrosive polyurethane coatings. Ind. Eng. Chem. Res. 2013, 52, 1562-1570. [CrossRef]

(C) 2018 by the authors. Licensee MDPI, Basel, Switzerland. This article is an open access article distributed under the terms and conditions of the Creative Commons Attribution (CC BY) license (http:// creativecommons.org/licenses/by/4.0/). 